Review Article

\title{
ANESTHESIA FOR DAY CARE SURGERY
}

\section{Anil Shetty ${ }^{1} \&$ Raveendra US}

${ }^{1}$ Associate Professor, ${ }^{2}$ Professor, Department of Anaesthesiology \& Critical Care,

K.S. Hegde M edical Academy, Nitte University, M angalore, Karnataka, India.

\section{Correspondence :}

Anil Shetty

Associate Professor, Department of Anaesthesiology \& Critical Care,

K.S. Hegde Medical Academy, Nitte University, Mangalore - 575 018, Karnataka, India.

M obile : +91 9448012303 E-mail : shetty.anil10@gmail.com

\section{Abstract:}

Day care procedures, diagnostic and therapeutic are increasingly popular, due to advances in anesthesia, surgery and medical technology. Carefully selected patients with optimized co morbid conditions, if any can be taken up for ambulatory surgery which includes sedation, regional and general anesthesia.

Careful selection of drugs and airway devices with a proper plan leads to success. Standard monitoring is mandatory .Complications should be anticipated with the goal of early recognition and management. Appropriate discharge criteria should be applied before sending the patient home. Backup facilities of inpatient treatment should be available

Keywords: Ambulatory anesthesia, location and facilities, newer drugs, postoperative nausea and vomiting, post anesthesia discharge score.

\section{Introduction :}

Recent advances in anesthetic and surgical practices have facilitated the rapid rise in ambulatory surgery throughout the world. With the availability of rapid, short acting anesthetic, analgesic, sympatholytic and muscle relaxant drugs, as well as improved monitoring devices, it has been possible to minimize the adverse effects of anesthesia on the recovery process.Improvements in the perioperative care of outpatients have allowed surgeons to perform an increasing array of more invasive surgical procedures on an ambulatorybasis. ${ }^{1}$

\section{Advantages of ambulatory anesthesia}

Ambulatory anesthesia can offer a number of advantages for patients. Outpatient surgery has been performed safely with a remarkably low incidence of both minor and major morbidity. M ain advantages are reduced risk of nosocomial

\begin{tabular}{|c|}
\hline Access this article online \\
\hline Quick Response Code \\
\hline
\end{tabular}

\section{Patient selection}

As with surgical procedures, the guidelines for patient selection have become much more liberal.The patients planned to undergo procedures should beASA I and II physical status. According to new guidelines, medically stable ASA III can also be taken up for ambulatory procedures. Among the pediatric group, all ages except premature infants, younger than 60 weeks post conception can be taken up. Several medical conditions and procedures previously considered as contraindications are now accepted if the ambulatory set up isappropriate and the medical conditions are optimized.These include patients with cardiac diseases, obstructive sleep apnea (OSA), some types of laparoscopic surgery.

\section{Contraindicationsfor day care procedures}

1. Emergency procedures

2. ASA III patients and above, with certain exceptions

3. Poor cardiac risk such as cardiac failure, significant arrhythmia

4. Severe aortic and mitral stenosis

5. Severe obstructive sleep apnea

6. Long duration and extensive procedures

7. Major laparoscopic surgery 


8. Need for blood transfusion
9. Need for postoperative ventilation
10. Anticipated sever postoperative pain requiring
intravenousanalgesics
11. Lack of hospital backup

\section{Facility Design and Safety}

Ambulatory surgical facilities need to be well designed to ensure efficient delivery of surgical services at the lowest possible cost.The ambulatory surgical units can be of fourbasic designs.

1. Hospital integrated, where it is part of regular operation theatre set up and ambulatory surgical patients are managed in the same surgical facility as inpatients. Outpatients may have separate preoperative preparation and recovery areas.

2. Hospital based,by beingwithin a hospital building or complex, but is separate from the main operation theatre and functionally independent.

3. Free standing,which is a completely independent or autonomous set up with its own preoperative, perioperative diagnostic and therapeutic facilities.

4. Office based. (Operating or diagnostic suites). Many of the diagnostic or therapeutic procedures like MRI, various system endoscopies and pain clinic procedures are done on an office based or stand alone ambulatory set up. ${ }^{2}$

\section{Location and facilities}

To be optimally efficient, it is recommended that allthe outpatient services be organized in one dedicated area.The waiting room, preoperative evaluation area, preanesthesia room, operating suites and recovery areas should be in close proximity so that surgeons and anesthesiologists are able to visit the patient and family before and after the operation without losing time in transit. Patient transportation time is also reduced to a minimum, and extra personnel are unnecessary because the circulating nurse can transport the patient to and from the operating room.Anesthesia and airway equipments should be appropriate for the goals of the centre in terms of the surgical procedures being planned.Staffs should be trained in patient care, including monitoring and resuscitation. They should be familiar with the surgical procedures conducted in the centre.Tie up with a hospital and facilities for transfer of patients in case of emergency is recommended. The policy and protocol should be written and easily accessible.

\section{Procedures suitable for ambulatory anesthesia.}

Dental- Extraction, restoration, certain facial fractures

Dermatology- Excision of skin lesions

General- Biopsy, endoscopy, excision of masses, hemorrhoiedectomy, herniorrhaphy and laparoscopic procedures

Pain clinic-nerve blocks, epidural injection.

Gynecology- dilatation and curettage, biopsy, hysteroscopy, and laparoscopic procedures

Ophthalmology- cataract extraction, chalazion excision, strabismus repair, and tonometry

Orthopedic- closed reduction, manipulation under anesthesia, carpel tunnel release, knee arthroscopy, shoulder reconstructions and minimally invasive hip replacements.

Otolaryngology- Adenoidectomy, laryngoscopy, mastoidectomy, myringotomy, polypectomy, rhinoplasty, tonsillectomy, and tympanoplasty.

Plastic surgery- cleft lip repair, liposuction, mammoplasty, otoplasty, scar revision and skin graft.

Urology- circumcision, cystoscopy, lithotripsy, prostate biopsy, and certain laparoscopic procedures

Radiology -Magnetic Resonance Imaging (MRI), Computerized Tomography (CT)

\section{Preoperative evaluation}

The primary objective of the preoperative assessment is to identify patients who have concurrent medical problems requiring further diagnostic evaluation or active treatment before elective surgery. Preexisting medical conditions such as hypertension, obesity, asthma and gastro esophageal reflux disease should be optimized before the proposed ambulatory procedures. Patients with specific 
anesthetic concerns (difficult airway, active cardiac disease) and those at increased risk for perioperative anesthetic and surgical complications should be identified in order to devise strategies to prevent postoperative complications.

Primary components of preoperative assessment are history, physical examination and laboratory testing, patients' medical history is clearly being the most valuable .History of sleep disturbances, snoring and day time sleepiness indicate the possibility of obstructive sleep apnea. Similarly, symptoms like stridor, hoarseness, reduction in effort tolerance etc should lead to further detailed evaluation. Clinical examination should focus on identifying changes in various organ systems particularly respiratory and cardiovascular system due to any coexistingillness.

Routine preoperative laboratory testing of patients before ambulatory surgery is unjustified and results in wastage of resources. ASA I patients, less than 40 years with normal airway and those not on any drug therapy may not require any investigations. ASA II patients, age more than 40 years, smoker and those who consume alcohol regularly may require investigations appropriate to the surgical procedures. Patients with an unexplained hemoglobin concentration of less than $10 \mathrm{~g} / \mathrm{dl}$ should be considered for further evaluation before elective out-patient surgery because a low hemoglobin level may be associated with diseases that could influence perioperative mortality and morbidity. ${ }^{3}$

\section{Preoperative preparation}

Optimal preoperative preparation of outpatients makes ambulatory surgery both safer and more acceptable for patients and hospital staff. The preparation process is aimed at reducing the risks inherent in ambulatory surgery, improving patient outcome and making the surgical experience more pleasant for the patient and family.

1. Fasting and premedication Standard fasting guidelines should be followed, avoiding excessive fasting.Unless outpatients have delayed gastric emptying (eg: diabetics), the requirement for a prolonged fasting is not justified. Adequate hydration( eg, allowing oral intake of clear fluids up to 2 to 3 hours before surgery ) is associated with a decreased incidence of postoperative side effects, including pain, dizziness, drowsiness, thirst and nausea.

2. Premedicationwith long acting sedatives like diazepam and lorazepam may be avoided.Midazolam, with its short duration of action and good recovery profile can be used for anxiolysis and for smooth separation from parents in case of pediatric patients. Routine prophylaxis with $\mathrm{H}_{2}$ blockers, proton pump inhibitors or prokinetic drugs is not indicated. However, those at risk of aspiration due to comorbid conditions should receive these drugs.

3. Preexisting medications should be continued or stopped depending on the patients' health condition.

4. Informed consent should be obtained.

\section{Basic Anesthetic Techniques}

Different anesthetic techniques and drugs have been used for ambulatory procedures. These can be performed under monitored anesthesia care (MAC), sedation, regional anesthesia or general anesthesia or a combination of more than one technique.

The main objective is to formulate an anesthetic plan, which while providing ideal intraoperative conditions, also ensures rapid recovery to enable same day discharge.Ideal conditions imply a calm and cooperative patient, easy separation from parents in case of children, smooth induction, and adequate analgesia, depth of anesthesia and muscle relaxation. ${ }^{4}$

\section{MAC and sedation}

The combination of local anesthesia, and /or peripheral nerve blocks withintravenous sedative and analgesic drugs is commonly referred to as MAC.Advantages are lack of significant effects on hemodynamics, avoidance of multiple drugs, avoidance of airway instrumentation and rapid recovery compared to general anesthesia. Disadvantages are potential for respiratory depression depending on level of sedation, risk of airway obstruction, 
oxygen desaturation and even aspiration.

The standard care for patients receiving M AC should be the same as for patients undergoing general or regional anesthesia and includes a standard preoperative assessment, intraoperative monitoring and postoperative recovery care.Vigilant monitoring is required because patients may rapidly progress from a light level of sedation to deep sedation and, thus may be at risk for airway obstruction, oxygen saturation and even aspiration. All necessary precautions like preparation for general anesthesia for management of failed sedation and complications related to sedation or procedure should be taken.

It is always safer to maintain sedation at minimal or moderate levels, in both of which the airway is reasonably well protected. Using single drug with specific objectives of sedation is highly recommended. Also the effect of the drug on airway reflexes, cardiovascular system and postoperative nausea and vomiting (PONV) should be kept in mind. Ideally propofol, fentanyl, midazolam and dexmedetomedine are the common and popular drugs used for sedation.

\section{Regional anesthesia (RA)}

Regional anesthesia can offer many advantages over general anesthesiawith respect to speed of early recovery. In addition to limiting the anesthetized area to the surgical site, common post-operative side effects of general anesthesia (nausea, vomiting, dizziness, lethargy) can be minimized.Simple regional anesthesia techniques (IVRA, peripheral nerve blocks) are preferreddue to the lower incidence of side effects, improved recovery profile and cost effectiveness.Spinal and epidural analgesia also can be used. Lignocaine is no more a choice of local anesthetic (LA) for central neuraxial blocks or for peripheral nerve blocks. Bupivacaine, in concentrations from 0.25 to $0.5 \%$ is most commonly used, though other LA like levobupivacaine and ropivacaine are also acceptable.Adjuvants like dexmeditomidine and fentanyl can be added to local anesthetics for RA.Use of ultrasound(USG)has become popular for nerve blocks. Advantages are reduced volume of injection, rapid onset of action and reduced risk of nerve injury.

\section{General anesthesia (GA)}

GA is indicated for many ambulatory procedures and has the advantages of safe and protected airway and control over the patient's physiology including ventilation while providing suitable conditions for surgery like muscle relaxation. The choice of drugs is discussed above. Any plan for GA should consider risk of postoperative nausea and its management. Volatile anesthetics, sevoflurane and desflurane are popular for ambulatory anesthesia, with or without nitrous oxide, because of easy titratability and rapid recovery, more so with desflurane. However the incidence of Postoperative nausea vomiting is more with volatile anesthetics,which is further increased by the addition of nitrous oxide.

Airway device is decided based on the surgical needs. Endotracheal intubation can be performed with succinylcholine or propofol - remifentanyl combination.A supraglottic airway device should always be considered as alternate to intubation.

\section{Drugs:}

Drugs used for ambulatory anesthesia should have shorter duration of action and be associated with complete recovery, with minimal adverse effects.

\section{Preferred drugs:}

\section{Premedicants:}

M idazolam ( $0.1-0.15 \mathrm{mg} / \mathrm{kg}$ ), is ideal for both adults and children. In children it facilitates smooth. separation from parents in less than 30 minutes. Anticholinergics such as atropine $(.01-.02 \mathrm{mg} / \mathrm{kg})$ or glycopyrrolate $(.005-.01 \mathrm{mg}$ $/ \mathrm{kg}$ ) have been used.

\section{Induction Agents:}

Propofol ( $25-50 \mathrm{mcg} / \mathrm{kg} / \mathrm{min}$ ) is the most commonly used drug for induction of anesthesia, maintence of sedation and treatment of nausea and vomiting. Thiopentone, Etomidate and Ketamine have been used, but now mostly replaced by Propofol. Etomidate $(0.2-0.3 \mathrm{mg} / \mathrm{kg})$ is 
preferred when hemodynamic stability is important.

\section{Analgesics}

Fentanyl (1 - 2mcg/kg) and remifentanyl (where available) are used for analgesia. Alfentanyl ( 15-30 $\mathrm{mcg} / \mathrm{kg}$ ) also has been used in ambulatory set up with favorable results.

Dexmedetomedine is emerging as the near ideal drug for sedation ( 1 - $2 \mathrm{mcg} / \mathrm{kg}$ bolus loading over 10 - 20 minutes followed by $0.25-0.5 \mathrm{mg} / \mathrm{kg} / \mathrm{h}$ for maintenance ). ${ }^{5}$

\section{Muscle relaxants:}

M ivacurium (0.15- $0.20 \mathrm{mg} / \mathrm{kg}$ ) ,atracurium ( 0.4 - 0.5 $\mathrm{mg} / \mathrm{kg}$ ) or rocuronium ( $0.6 \mathrm{mg} / \mathrm{kg}$ ) are used for muscle relaxation. Succinylcholine, $(1 \mathrm{mg} / \mathrm{kg})$ though associated with postoperative myalgia, has been safely used for to facilitate intubation.

\section{Reversal Agents:}

Neostigmine (.04 mg / kg) and glycopyrrolate combination is used for reversal. Alternately, sugammadex can be used. Use of neostigmine is associated with higher incidence of PONV than sugammadex.

\section{Newer drugsfor day care surgery}

Modifications in the formulations of existing drugs and research have resulted in introduction of newdrugs into clinical practice or are undergoing clinical trials. They are

1. Modifications of popofol, by increasing the concentration of the the drug, creating emulsions with less than $10 \%$ oil, emulsions having different fatty acid contents and modification of emulsion droplets with proteins.

2. Remimazolam, ultra short acting, highly selective GABA agonist benzodiazepine. It has both rapid onset and offset compared to midazolam.

3. Etomidate derivatives like methoxycarbonyl etomidate and cyclopropyl etomidate.

4. Gantacurium and CW 002are the two muscle relaxants under investigations.

5. Non intravenous fentanyl preparations like Staccato fentanyl for Inhalation which delivers aerosolized fentanyl through a single metered inhalation.
6. S ketamine, an isomer of ketamine without hallucination is already available for clinical practice and yet to be introduced in India.

7. Neurokinin - 1 receptor antagonists like apripitent (40 mg orally), casopritent and rolapitant are the recently introduced antiemetic agents.

8. EXPAREL, bupivacaine liposome injectable suspension $1.3 \%$, for surgical site infiltration

9. SABER Bupivacaine is an extended release bupivacaine hydrochloride formation.

10.Transdermal scopolamine patches are useful in prevention of PONV.

\section{Monitoring duringanesthesia}

Minimum mandatory standards of monitoring include using pulse oximetry,non-invasive blood pressure and electrocardiogram (ECG) in all patients and $\mathrm{ETCO}_{2}$ in intubated patients.Additional monitors include neuromuscular blockade monitor, temperature and depth of anesthesia monitor (particularly for patients with history of awareness).In addition to this a nonanesthesia staff familiar with anesthetic procedures and equipment must be present and vigilant. The staff should be trained in postprocedure observation and resuscitation.

\section{Postoperative Management}

PONV and pain are the two problems in the postoperative period which have the potential to delay the discharge and also affect the quality of ambulatory anesthetic care.The incidence of emesis is low in infants, gradually increases towards adulthood, and then decreases at advanced age. Choosing an anesthetic plan with drugs like propofol which have inherent antiemetic properties plays an important role in preventing PONV. Nevertheless antiemetic drugs belonging to different groups such as $5 \mathrm{HT} 3$ receptor antagonists, (ondensetron 4- $8 \mathrm{mg} \mathrm{IV}$, palanosetron $0.075 \mathrm{mg}$ IV and granisetron0.35- $1.5 \mathrm{mgIV}$ ), phenothiazines, (Prochlorperazine 5- $10 \mathrm{mg}$ IM/IV), anticholinergics, such as transdermal scopolamine patch, corticosteroids(dexamethasone 4-5mg IV) and neurokinin- 
1 antagonists like aprepitant 40mg orallyhave been used.

Pain management is critical for improving the quality of anesthetic care and to reduce morbidity in the postoperative period. Optimizing pain management is necessary to maximize the benefits of ambulatory surgery for both patients and health care providers. Pain management consists of identifying factors which are associated with higher risk of severe postoperative pain such as age, gender, duration and type of surgery. Planning for multimodal analgesia (M M A) is the most effective way of managing pain. M MA involves use of different groups of analgesic drugs by more than one route and use of more than one technique of aneathesia. An example is use of RA and opiod analgesic for intraoperative pain management followed by wound infiltration, use of nonsteroidal antiinflammatory analgesics ( NSAID) and paracetamol (acetaminophen ). Although opiod analgesics play an important role in the management of pain, the adjunctive use of nonopiod analgesics will probably assume a greater role in the future. ${ }^{7}$

\section{Risk factors for PONV}

\begin{tabular}{|l|}
\hline Patient related factors \\
\hline Age \\
\hline Gender \\
\hline History of motion sickness \\
\hline Pre-existing diseases \\
\hline Anesthesia related factors \\
\hline Premedication \\
\hline Opiod analgisics \\
\hline Induction and maintenance anesthetics \\
\hline Inadequate hydration \\
\hline Surgery related factors \\
\hline Length of surgery \\
\hline Operative procedure \\
\hline
\end{tabular}

White pf: postoperative nausea and vomiting; its etiology, treatment and prevention, anaesthesiology 77:162, 1992

\section{Discharge criteria}

The three stages of recovery after ambulatory surgery are the early, intermediate, and late recovery phases. The early and intermediate recovery stages occur in the ambulatory or office based surgical facility, whereas late recovery refers to the resumption of normal daily activities and occurs after the patient has been discharged home.
Early recovery is the time interval during which patients emerge from anesthesia, recover control of their protective reflexes, and resume early motor activity. During this phase of recovery, patients are cared for in the PACU and day surgery unit, where their vital signs and oxygen saturation are carefully monitored and supplemental oxygen, analgesics, or antiemetic'sare administered as necessary. ${ }^{8}$

The modified Aldrete or white score is commonly used to assess the readiness of patients to be transferred to the day surgery recovery area. It assesses the activity, respiration, circulation, consciousness and oxygen saturation, each given a score of 2, 1 and 0 total maximum score being 10 . Patient should be discharged to step down unit only after the score is more than or equal to $9 .{ }^{9}$

During the intermediate recovery period, patients progressively begin to ambulate, drink fluids, void and prepare for discharge. The choice of anesthetic technique, as well as the appropriate use of postoperative analgesic, and antiemetic drugs, all have an impact on the duration of the intermediate recovery period.

The late recovery period starts when the patient is discharged home and continues until complete functional recovery is achieved and the patient is able to resume normal activities of daily living.

Guidelines for safe discharge from an ambulatory surgical facility include stable vital signs, return to baseline orientation, ambulation without dizziness, minimal pain and PONV, and minimal bleeding at the surgical site. ${ }^{10}$

A revised post anesthesia discharge scoring system (PADS) is available for discharge from the hospital or ambulatory unit to home. Unlike the modified Aldrete's scoring system, PADS includes activity, nausea and vomiting, pain and surgical bleeding, in addition to vital signs as parameters with a total score of 10. Patient is discharged only after achieving a score of 9 or more. 


\begin{tabular}{|l|l|}
\hline \multicolumn{2}{|l|}{ Postanesthesia discharge scoring system ( PADSS ) } \\
\hline Vital signs & within $20 \%$ of preoperative value \\
2 & $20 \%-40 \%$ of preoperative value \\
1 & $40 \%$ of preoperative value \\
\hline & \\
\hline Activity, mental status & \\
2 & Oriented and steady gait \\
1 & Oriented or steady gait \\
0 & Neither \\
\hline Pain, nausea, vomiting & \\
2 & M inimal \\
1 & M oderate \\
0 & Severe \\
\hline Surgical bleeding & \\
2 & Minimal \\
1 & M oderate \\
0 & Severe \\
\hline Intake and output & PO fluids and voided \\
2 & PO fluidsor voided \\
1 & Neither \\
\hline
\end{tabular}

Frances chung,Recovery pattern and home readiness after ambulatory surgery,Anesth Analg - 1995:80:896-902

\section{Conclusion:}

The number of patients undergoing anaesthesia on a day care basis has tremendously increased due to availability of

\section{References}

1 White PF. Ambulatory anesthesia advances into the new millennium. Anesth Analg 2000,90.1234-5

2 Mathew R. EngAmbulatory anesthesia.2010,78.2420-24

3 Ford MK. Prediction of perioperative cardiac complications and mortality by the revised cardiac risk index. A systemic review. Ann Intern M ed 2010,6.467-72

4 Liu SS, Strodtbeck WM,Richman JM,et al. A comparison of regional versus general anesthesia for ambulatory anesthesia. Anesth Analg 2005, 101.1634-42

5 Beverly K Philip,Frances Chung,Thomas j Conahan, et al. Remifentanyl compared with alfentanyl for ambulatory surgery using total intravenous anesthesia. Anesth Analg 1997,84.515-21

6 Anesthesiology Clinics.Ambulatory anesthesia June 2014. Volume 32, Number2

7 Paul F. The role of non opiod analgesic techniques in the management of pain after ambulatory surgery. Anesth Analg 2002,94.577-85

8 Frances chung, Recovery pattern and home readiness after ambulatory surgery, Anesth Analg 1995,80.896-902

9 Aldrete JA.The post anesthetic recovery score revisited.J Clin Anesth 1995,7.89-91

10 Frances chung, Postoperative pain in ambulatory surgery,Anesth Analg 1997,85.808-16 better drugs and better medical facilities. The procedures may be therapeutic or diagnostic and the set up hospital based, stand alone or office based. ASA I and II physical status patients are ideal for ambulatory anaesthesia and careful preoperative evaluation should lead to identification of serious medical, surgical and anaesthetic problems.

Different types of anaesthetic, MAC, regional or general anaesthesia can be chosen. Short acting drugs are preferred. Supraglottic airway devises are increasingly being used. Careful monitoring in the perioperative period is very important and should be continued till discharge. Newer drugs with still better recovery and safety profile compared to existing ones have contributed to further improvement in safety.

PONV and pain are the most important complications in postoperative period. Multimodal analgesia reduces pain significantly. Facilities for management of unanticipated complications should be a part of any ambulatory set up. 